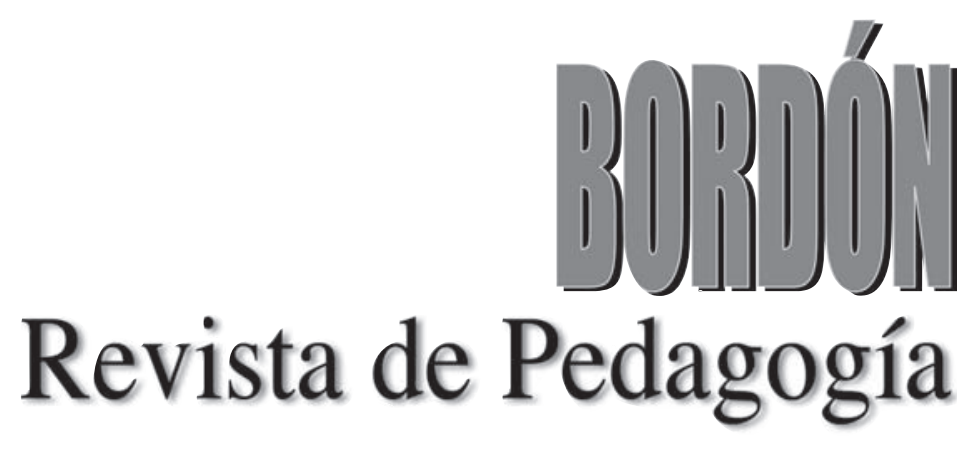

\author{
NÚMERO MONOGRÁFICO
}

El sistema educativo español: viejos problemas, nuevas miradas. Conmemoración de un bicentenario (1813-2013) María del Mar Pozo Andrés (coord.)

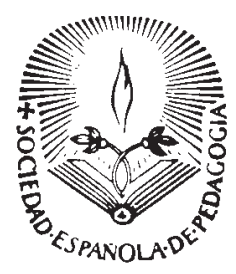





\title{
POLÍTICAS EDUCATIUAS Y PRÁCTICAS ESCOLARES: LA APLICACIÓn DE LA LEY DE ENSEñAIZZA PRImARIA DE 1945 En LAS AULAS ${ }^{1}$
}

\section{Educational politics and school practices: the implementation of the 1945 Primary Education Act in the classrooms}

\author{
MARÍA DEL MAR DEL POZO ANDRÉS \\ Universidad de Alcalá de Henares \\ TERESA RABAZAS ROMERO \\ Universidad Complutense de Madrid
}

DOI: 10.13042/Bordon.2013.65408

INTRODUCCIÓN. Este artículo analiza la influencia de la política educativa en la realidad escolar del primer franquismo. Se ha estudiado la influencia en la práctica educativa de los años cincuenta de algunos principios educativos reflejados en la Ley de Enseñanza Primaria de 1945 y en los Cuestionarios Nacionales para la enseñanza primaria aprobados en 1953. MÉTODO. Se ha combinado el método histórico-educativo para el análisis de la política educativa y el método etnográfico para la interpretación de las prácticas escolares. La principal fuente de documentación ha sido la colección de memorias pedagógicas conservadas en el Fondo Anselmo Romero Marín del Museo M. B. Cossío de la Universidad Complutense. RESULTADOS. El análisis realizado permite detectar ciertas continuidades con los planteamientos curriculares de periodos históricos anteriores y con las metodologías renovadoras de los años treinta. Por otra parte, no parece que la legislación educativa del primer franquismo estuviera muy presente en la cotidianeidad escolar, salvo en los aspectos de carácter simbólico y ritualizado. Lo que nos lleva a confirmar que la historia de la escuela se va construyendo con la cultura escolar empírica que desarrollan los docentes y no se aprecian saltos significativos, a pesar de los giros ideológicos que pueda tener la política educativa. DISCUSIÓN. Se podría introducir en el debate historiográfico sobre el franquismo el modelo de la "gramática de la escuela" que desarrollaron hace ya varios años Tyack y Cuban. La discusión habría de centrarse en hasta qué punto la política educativa del franquismo supuso una ruptura curricular y metodológica con la etapa anterior. De alguna forma se puede interpretar que las prácticas docentes son inmunes a los cambios políticos y dependen de la mentalidad y de las dinámicas profesionales de los maestros y maestras.

Palabras clave: Enseñanza primaria, Franquismo, Ley de 1945, Política educativa. 


\section{Introducción: nuevos interrogantes sobre la Ley de Educación Primaria de 1945}

Una de las manifestaciones más conocidas de la política pedagógica del franquismo fue la Ley de Educación Primaria promulgada el 17 de julio de 1945 y que estuvo vigente durante veinte años, hasta su sustitución por la Ley de 21 de diciembre de 1965. Por ello puede ser considerada no solo como una de las normativas legales sobre enseñanza más influyentes de este régimen político, sino también como una de las legislaciones educativas de más largo recorrido en el siglo XX.

Por ser un tema suficientemente conocido y tratado exhaustivamente en la historiografía educativa reciente, no nos vamos a detener en explicar las implicaciones políticas e ideológicas que trajo consigo el nacional-catolicismo. Todos los historiadores han coincidido en señalar que el franquismo intentó propagar su ideario religioso y patriótico a través de la educación. El amplio cuerpo de normas y circulares publicadas desde 1937 en la denominada "zona nacional" durante la Guerra Civil y enriquecido en los primeros años cuarenta, se reorganizó y sistematizó en la Ley de 17 de julio de 1945, dirigida específicamente a la enseñanza primaria, en la cual se formularon y resaltaron los valores religiosos y nacionales que debían regular la educación de los españoles (Puelles, 1999).

Esta Ley de 1945 fue la primera que estableció una "declaración de principios", enunciando los fines de la enseñanza primaria. Pretendía proveer a las jóvenes generaciones con una formación del carácter "en orden al cumplimiento del deber y a su destino eterno", e infundirles "el amor y la idea del servicio a la Patria, de acuerdo con los principios inspiradores del Movimiento". Los artículos 5 al 11 establecieron las características fundamentales de esta educación primaria, que serían las siguientes: adecuación a los principios del dogma y la moral católica, formación de un espíritu nacional "fuerte y unido", que despertase "la alegría y el orgullo de la Patria", obligación de utilizar el castellano como "lengua nacional" en toda la enseñanza primaria, fomento de la educación social, es decir, de los hábitos necesarios para impulsar la convivencia humana, desarrollo de los conocimientos intelectuales mediante métodos que, "sin olvidar la tradición pedagógica española", se adaptasen "a las exigencias científicas que plantea la pedagogía moderna", potenciación de la educación física "para formar una juventud fuerte, sana, disciplinada" y preparación "para la superior formación intelectual o para la vida profesional del trabajo en la industria y el comercio o en las actividades agrícolas", en el caso de los varones, pues a las féminas se las capacitaría específicamente "para la vida del hogar, artesanía e industrias domésticas" (Ley, 1945: 387-388).

Estos objetivos tuvieron su plasmación curricular en un plan de materias que se estructuró en tres ámbitos con distinto orden de importancia: materias instrumentales, que crearían los hábitos necesarios para el aprendizaje de otros contenidos, y que incluían la Lectura interpretativa, la Expresión gráfica -Escritura, Ortografía, Redacción y Dibujo- y el Cálculo; materias formativas, que constituían el core de la educación intelectual y moral, abarcando la Religión, la Formación del Espíritu Nacional -que englobaba también la Geografía e Historia-, la Lengua nacional, las Matemáticas y la Educación Física; y materias complementarias, destinadas a completar la cultura mínima primaria, en las que se integraron las Ciencias de la Naturaleza, los contenidos artísticos -Música, Canto y Dibujo-y los de carácter práctico —Trabajos Manuales y Labores femeninas-, así como "la adquisición de hábitos activos para la educación social" del alumnado (Ley, 1945: 394). Sutilmente, el legislador resaltó la importancia de las materias consideradas formativas, que contemplaba y resaltaba la formación política y religiosa de los alumnos, 
una formación que, a priori, debía ocupar una buena parte del horario escolar total.

$\mathrm{Y}$, no solamente se definieron claramente los objetivos y contenidos de la enseñanza primaria, sino que también se anunció, en el artículo 38, la publicación de unos cuestionarios que recogerían los contenidos mínimos de cada materia, así como los ejercicios y actividades en los que se plasmaría la labor del alumno. Además, se estableció un mecanismo de comprobación de la labor escolar, medida en términos de resultados, a través de un sistema de pruebas objetivas y otros procedimientos de evaluación que regularía el Ministerio de Educación Nacional. Como puede percibirse, el objetivo último de la política pedagógica franquista con esta ley era dotar de una uniformidad absoluta, tanto en contenidos como en valores, a toda la enseñanza primaria española, convirtiéndola en un poderoso medio de expansión y afianzamiento de la ideología del régimen.

La historiografía contemporánea de la educación parece haber asumido la hipótesis de la uniformidad en cuanto a contenidos, métodos y valores que tuvo la enseñanza primaria española durante los primeros años del franquismo. El objetivo de este artículo es, precisamente, estudiar la influencia de la política pedagógica en la realidad escolar de los años cincuenta, a través del análisis de algunos principios educativos reflejados en la Ley de Educación Primaria de 1945 y su concreción curricular en los Cuestionarios Nacionales para esa etapa, aprobados en 1953. A partir de este planteamiento general, nos hemos formulado dos interrogantes:

1. Las escuelas primarias españolas de los años cincuenta, ¿reflejaron la homogeneidad curricular que persiguió la Ley de Enseñanza Primaria de 1945?

2. ¿Es posible encontrar continuidades con las tradiciones curriculares y metodológicas renovadoras implantadas en los años veinte y treinta?

\section{El desarrollo de la Ley de Educación Primaria de 1945: los Cuestionarios Nacionales de Enseñanza Primaria de 1953}

Los docentes españoles venían demandando la publicación de cuestionarios y programas con unos contenidos comunes para todas las escuelas españolas desde la publicación del plan de estudios para la enseñanza primaria de 1901. Haciéndose eco de estas demandas, algunos inspectores provinciales divulgaron diseños curriculares con anterioridad y posterioridad a la promulgación de la Ley de 1945 (López del Castillo, 2013). Pero habría que esperar ocho años y un cambio en la cartera ministerial para que se publicaran los primeros Cuestionarios Nacionales destinados al nivel de enseñanza primaria. Sin embargo, no faltaron iniciativas, bien que fallidas.

Entre los antecedentes debemos mencionar los programas de 1938 estudiados por Peralta Ortiz (1996) y por López Bausela (2012), que nunca fueron publicados debido a las distintas orientaciones políticas mantenidas en el seno del gobierno franquista. La comisión nombrada por el ministro de Educación Nacional, Pedro Sainz Rodríguez, pretendió incorporar algunas de las innovaciones pedagógicas introducidas por el movimiento de la Escuela Nueva, respetando los valores ideológicos y políticos del nuevo régimen, por lo que podría interpretarse que se produjo un intento de establecer cierta continuidad con la renovación educativa de la etapa anterior. No obstante, el estudio crítico realizado por López Bausela pone en entredicho que estas innovaciones respondiesen a una aparente modernidad, puesto que a lo largo de los programas se aprecia una "única y exclusiva finalidad: educar al alumnado en el amor a Dios y a la Patria" (López Bausela, 2012: 46).

Nuestro análisis de los Cuestionarios Nacionales de 1953 también desvela algunas singularidades que requieren que nos detengamos en su análisis. Fueron aprobados bajo el mandato del 
ministro Ruiz-Giménez y elaborados por una comisión de inspectores liderada por Adolfo Maíllo. En su interesante biografía sobre este educador, Juan Mainer y Julio Mateos le definen como un pedagogo orgánico del Estado muy peculiar y controvertido (Mainer y Mateos, 2011: 15-16). Adolfo Maíllo desempeñó cargos de responsabilidad pública en el periodo republicano y en el franquista. Gran conocedor de la escuela como maestro y, posteriormente, como inspector, fue sobre todo un defensor de la escuela pública (Mainer y Mateos, 2011: 18). Él había sido uno de los inspectores provinciales que redactó orientaciones curriculares, elaborando las de su zona de inspección, en la provincia de Salamanca, en el año 1944. Él desveló también el origen inmediato de los Cuestionarios Nacionales de 1953, pues cuando el equipo de inspectores centrales tomó posesión de su cargo, plantó inmediatamente al director general de Enseñanza Primaria y al ministro Ruiz Giménez el proyecto de redactar unas directrices para orientar "las tareas escolares en un sentido didácticamente eficaz, dando, por otra parte, unidad de actuación a las escuelas, sin perjuicio de que cada inspector permitiese a los maestros de su zona reflejar en su trabajo diario la propia personalidad" (Maíllo, 1989: 316). Dada la dilatada experiencia de los autores en esta cuestión, y una vez que el ministro les dio luz verde para abordar la empresa, necesitaron tan solo dos meses para su redacción.

Un estudio de estos primeros Cuestionarios Nacionales muestra ciertas paradojas que nos gustaría señalar. En el discurso pedagógico de los Cuestionarios se dejan entrever algunas continuidades innovadoras con la pedagogía activa, generalizada durante la etapa republicana, tal y como se refleja en el preámbulo:

"La enseñanza toda será concreta, viva y activa. Partirá del ambiente próximo, enlazando con sus realidades cada lección y cada ejercicio, y volverá al área de lo inmediato, lo conocido o lo deseado —-formas diversas de inmediatez-, para insertar en ella las ideas deducidas del ejercicio o la lección de que en cada caso se trate. Esto vale tanto como decir que debe huirse de todo verbalismo, de todo memorismo, de toda divagación por los campos de lo espectralmente alejado de la órbita de realidades en que se mueven los intereses y afanes del niño" (Ministerio de Educación Nacional, 1953: 10).

Resulta chocante que, en un esfuerzo para distanciarse de los pedagogos de la Escuela Nueva, se justificasen los argumentos sobre la escuela vitalista, intuitiva y centrada en los intereses del niño, con el recurso a autores clásicos como Séneca. Esta paradoja se puede entender si tenemos en cuenta el pensamiento de Adolfo Maíllo, quien parece fue el que se encargó de redactar las orientaciones didácticas expuestas en el preámbulo. Como han puesto de manifiesto sus biógrafos, este pedagogo participó del reformismo pedagógico de la Escuela Nueva, conformando su ideario educativo autores como Claparède, Kerschensteiner y Dewey (Mainer y Mateos, 2011: 40). No obstante, se aprecian rupturas en su pensamiento pedagógico, que pueden entenderse como resultado de un esfuerzo para adaptarse a los posicionamientos ideológicos del primer franquismo. Y posiblemente este esfuerzo de adaptación estuvo muy presente también en la redacción de los Cuestionarios, pues sus autores, casi todos ellos inspectores formados en la etapa previa a la Guerra Civil y en una cultura pedagógica dominada por el movimiento de la Escuela Nueva, intentarían encajar los avances metodológicos desarrollados con anterioridad en el contexto ideológico del franquismo, con el fin de conseguir una aprobación de estos programas que finalmente lograron. También cabe explicar el carácter innovador de dichos Cuestionarios por la política educativa integradora o conciliadora que siguió el ministro que los refrendó, Joaquín Ruiz Giménez. Este delegó en una comisión de expertos o profesionales de la educación la elaboración de los contenidos curriculares de la 
Políticas educativas y prácticas escolares: la aplicación de la Ley de Enseñanza Primaria de 1945 en las aulas

enseñanza primaria, apostando por realizar una reforma más técnica que ideológica.

La estructura interna que presentan los Cuestionarios responde al currículo diseñado en la Ley de Educación Primaria de 1945. Es curioso constatar que la elaboración de los programas de la mayor parte de las asignaturas formativas y complementarias (Formación del Espíritu Nacional, Educación Física, Labores, Economía Doméstica, Música, Higiene) no se consideró competencia de la Comisión, sino que fue encomendada a las instituciones sustentantes del régimen, como eran la Falange y la Sección Femenina, mientras que de los de Religión se responsabilizó la jerarquía eclesiástica. Por lo tanto, los inspectores proporcionaron las orientaciones didácticas de las materias puramente instructivas o intelectuales como Lengua, Matemáticas, Geografía, Historia de España y Ciencias de la Naturaleza. Merece la pena detenerse en algunas cuestiones didácticas que se sugirieron para la enseñanza de estas materias.

En primer lugar, a nivel general, se aprecia una crítica constante al aprendizaje memorístico, teórico o verbal en todas las asignaturas, planteándose metodologías más activas e intuitivas que conectaban directamente con la renovación pedagógica del primer tercio del siglo XX. A modo de ejemplo, para la enseñanza de la Lengua se propuso que los estudiantes dialogasen o realizasen charlas sobre temas de su interés, acompañadas de actividades como la dramatización o ejercicios prácticos. Para las materias de Geografía y de las Ciencias de la Naturaleza, se recomendó la observación de la realidad física y del entorno natural más próximo al estudiante por medio de excursiones y paseos. Se sugirió el dibujo y los trabajos manuales de modelado para la representación de mapas geográficos, actividad característica de la pedagogía manjoniana y propiciada desde los manuales de Pedagogía de la época (Berrio, Rabazas y Ramos, 2006: 133), pero que también había sido considerada como una innovación en la enseñanza madrileña de los años treinta. Se invitó a los alumnos a que realizasen cuadernos de campo donde recogiesen sus observaciones, acompañadas de dibujos que ilustrasen el objeto de estudio. Para las escuelas urbanas se aconsejaron excursiones al campo y actividades de experimentación en la escuela, tales como el cultivo de macetas. Otra de las innovaciones que se introdujeron para la enseñanza de la Física fue la construcción de aparatos mediante el reciclado de materiales usados, siguiendo el concepto novedoso de la teoría pedagógica de la autoconstrucción del material (Rabazas, Ramos y Ruiz, 2009: 286).

En la enseñanza de la Historia, Adolfo Maíllo propuso un aprendizaje significativo basado en lecturas o biografías de personajes célebres, visitas a museos y monumentos, y evocaciones y dramatizaciones para recrear los acontecimientos más relevantes de la historia de España. Según Raimundo Cuesta, algunas de estas orientaciones didácticas pueden llevarnos a suponer que Maíllo se adelantó al narrativismo historiográfico actual, aunque manteniendo ciertos sesgos ideológicos herederos del franquismo (Cuesta, 1997: 160).

Los Cuestionarios llegaron a tales niveles de concreción que especificaban a los maestros cómo debían desarrollar sus clases. Introdujeron un nuevo concepto -denominado "orgánico"- de la lección, que consistía en transformar esta en "un conjunto de actividades de colaboración entre maestros y niños que, comenzando por una preparación, tanto de estos como de aquel, se continúa con un diálogo, lleno de dinamismo y de amenidad, en el que se intercalan acciones -ilustrativas, corroborativas, sugestivas- y termina con una serie, todo lo variada que se pueda, de ejercicios de aplicación (manualizaciones o ejercicios prácticos)" (Ministerio de Educación Nacional, 1953: 13). Una atención tan especial a la preparación de las lecciones demuestra que los autores de los Cuestionarios sabían perfectamente que gran parte de las actividades dentro del aula giraban en torno a ellas y que 
en la mayoría de las escuelas constituían un reducto del aprendizaje memorístico (Pozo y Rabazas, 2011: 112). En los próximos apartados analizaremos si estas palabras llegaron a calar y a cambiar la realidad escolar cotidiana de las aulas franquistas.

\section{El Fondo Romero Marín: una fuente excepcional para estudiar la práctica educativa en el franquismo}

La principal fuente de documentación que hemos utilizado en nuestro trabajo ha sido la colección de memorias de prácticas pedagógicas conservadas en uno de los fondos del Museo Manuel Bartolomé Cossío de la Universidad Complutense. Este acervo documental recibe el nombre de uno de los catedráticos de la Sección de Pedagogía de la Universidad Complutense de Madrid, Anselmo Romero Marín, que se encargó de dirigir las prácticas pedagógicas desde el año 1949 hasta mediados de los años setenta.

Dichas memorias constituyen una fuente excepcional para estudiar la realidad escolar del franquismo, tal y como han puesto de manifiesto diversas publicaciones (Martínez Navarro, 1997: 305-318; Pozo Andrés y Rabazas Romero, 2010: 165-194 y 2011: 99-120; Colmenar Orzaes, 2012: 197-210; Pericacho Gómez, 2012: 311-322; Poveda Sanz y Rabazas Romero, 2012: 323-336, y Ramos Zamora, 2012: 337-354). Estos trabajos se presentaban como monografías que describían de la forma más completa y objetiva posible una experiencia educativa, aportando una información exhaustiva sobre diferentes instituciones ubicadas en gran parte de la geografía española.

Del total de las 891 memorias conservadas en este archivo, para este artículo hemos seleccionado 135 , precisamente todas las presentadas entre 1950 y 1956, que hemos clasificado en dos periodos: las realizadas con anterioridad a la publicación de los Cuestionarios de 1953 (primera etapa, de 1950-1953) y con posterioridad a su promulgación (segunda etapa, de 1954-1956). La muestra seleccionada es altamente representativa de la situación escolar española, pues recoge monografías sobre escuelas de cincuenta provincias diferentes. Tal riqueza fue posible debido a la heterogeneidad del alumnado, que solo podía estudiar la especialidad de Pedagogía en la Universidad de Madrid. En estas memorias podemos encontrar información sobre una amplia variedad de escuelas primarias, que hemos agrupado en tres tipos:

1. Las escuelas públicas rurales, temática de la gran mayoría de memorias (79\%). Eran instituciones ubicadas en pueblos dispersos por toda la geografía española, casi siempre organizadas pedagógicamente como escuelas mixtas y unitarias, aunque también se estudiaron algunos grupos escolares graduados, generalmente de reciente construcción. Los autores de estas monografías eligieron estos centros porque eran docentes de las propias escuelas, o realizaron en ellas sus prácticas cuando estuvieron formándose como estudiantes de magisterio o asistieron a ellas como alumnos en su niñez, por residir en esos pueblos.

2. Las escuelas urbanas, habitualmente localizadas en Madrid, o en alguna otra ciudad relevante como Valencia, se estudian en un $15 \%$ de memorias. Eran instituciones que se ubicaban, o bien en el centro de la ciudad, o en algunos de los barrios suburbiales y poblados por las clases humildes. En este grupo podemos encontrar colegios públicos y privados, pertenecientes estos últimos a las congregaciones religiosas dedicadas a la enseñanza. Una de las posibles razones para escoger como objeto de estudio algunas de estas instituciones fue su mayor accesibilidad para los autores de las memorias, bien por cercanía a su vivienda, o por pertenecer a la misma orden religiosa que dirigía el colegio, o por ser docente o antiguo alumno del mismo. 
Políticas educativas y prácticas escolares: la aplicación de la Ley de Enseñanza Primaria de 1945 en las aulas

3. Los centros especiales o singulares, que hacen referencia a establecimientos de educación especial, internados de carácter social (reformatorios, orfelinatos) e instituciones de formación profesional. Este grupo representa el $6 \%$ de la muestra total, pero solo hemos considerado para nuestro estudio a aquellos que tenían clases de enseñanza primaria. Los estudiantes que se interesaban por este tipo de centros lo hacían, o bien porque eran docentes en ellos, o bien porque en su infancia o adolescencia habían sido alumnos de colegios similares.

\section{La Ley de Educación Primaria de 1945 en las aulas: aspectos curriculares}

Los estudiantes de Pedagogía apenas citaban en sus monografías la Ley de Educación Primaria de 1945, salvo como un referente muy lejano que, desde las alturas, marcaba los destinos de la escuela española de forma vaga e imprecisa. Ahora bien, es posible encontrar algunas diferencias entre los trabajos dedicados a la escuela privada y a la pública. Los que analizaban centros de congregaciones religiosas se manifestaban habitualmente bastante entusiastas de la ley, por su "espíritu católico", y reproducían todo el articulado en el que se reconocía "la primacía y plenitud del derecho docente de la Iglesia" (Navamuel, 1954: 21; López Chillón y García de Leza, 1953: 116), es decir, que tomaban la legislación como punto de partida y justificación de su actuación educativa. Sin embargo, los que estudiaban las escuelas públicas utilizaban la ley para demostrar su incumplimiento en los pueblos estudiados, especialmente en lo referente a la creación de escuelas, a las construcciones escolares y a la dotación de material pedagógico, argumentando que, si bien la legislación facilitaba la edificación de centros, el proyecto se realizaba con lentitud "y la obra... quizá nuestros nietos disfruten de ella porque los actuales escolares creo que no" (Galán Martínez, 1953: 96). Aunque se procuraba desviar las críticas desde la ley hasta los encargados de ponerla en práctica, no faltaron memorias que subrayaron directamente la cicatería y escasas expectativas de una normativa legal que funcionaba con unos cómputos poblacionales requeridos para crear escuelas más altos que los de principios de siglo, dándose la paradoja de que, en 1950, se consideraba que un mismo número de habitantes solo necesitaba la mitad de aulas escolares que en 1909 (Selles Vercher, 1956).

Este es, precisamente, el primer aspecto que sorprende al acercarse a las memorias de prácticas. Si bien es verdad que la mayoría de sus autores eran respetuosos e, incluso, entusiastas con la política educativa del régimen franquista, no faltaban voces que, con sutileza y discreción, manifestaban las dificultades de poner en práctica el articulado de la Ley de 1945. Y, además, estas voces discrepaban entre sí, haciéndonos intuir la presencia de un pensamiento divergente muy alejado del pensamiento único asociado tradicionalmente con el franquismo.

Cabe plantearse si esta divergencia de criterios puede trasladarse al diseño de materias establecido en la Ley de Educación Primaria de 1945 y si realmente se produjo la uniformidad curricular absoluta que el legislador perseguía. $\mathrm{Y}$, efectivamente, las memorias pedagógicas nos muestran una realidad curricular bastante diversificada. Solo dos escuelas de todas las estudiadas, la de Lucena del Cid y la del Santo Ángel de Vallecas, organizaban el cuadro de asignaturas de acuerdo con la ley de 1945, diferenciando entre instrumentales, formativas y complementarias, y es posible que, en el primer caso, esta distribución no fuera real sino una construcción de la autora de la memoria, quien también copiaba párrafos del preámbulo de los Cuestionarios Nacionales de Enseñanza Primaria (Beltrán Escrig, 1956: s.p., y Yerro Mainar, 1956: 79). Muchas escuelas impartían una representación reducida del currículum de 1945, obviando varias de las materias artísticas 
y la Educación Física. Pero un porcentaje elevado de centros ofrecía un abanico curricular mucho más cercano al plan de estudios de 1901 que al de 1945, incluyendo asignaturas presentes en el primero, pero no en el segundo, como Fisiología e Higiene, Ciencias Físicas, Químicas y Naturales, Derecho o Geometría. Y otros muchos, tanto públicos como privados, recogían contenidos que, o bien provenían de diseños curriculares de tiempos pretéritos, como las nociones de Agricultura, Industria y Comercio establecidas en la Ley Moyano de 1857, o bien jamás habían formado parte de ningún plan de estudios de carácter oficial, como la Urbanidad. Esta asignatura, sin ninguna tradición en la legislación española, era muy popular en las escuelas primarias, identificándose con la formación social prevista en el artículo 8 de la Ley de 1945, y se consideraba imprescindible por la propia idiosincrasia de la población asistente a las escuelas públicas y privadas en el mundo rural y suburbial, pues "estos niños, de gente pobre en su mayoría, no verán muy buenos modales en su hogar" (Bernal, 1953: 42).

La Ley de 1945 estableció dos grandes pilares axiológicos en la enseñanza primaria: el espíritu religioso, canalizado a través de la obediencia y el cumplimiento de todos los preceptos de la Iglesia católica, y el amor a la patria, expresado mediante el respeto hacia todas las normas e instituciones del Movimiento. Aunque ambos valores debían impregnar la vida entera de las escuelas, se plasmaban especialmente en dos asignaturas específicas de Religión y Formación del Espíritu Nacional. No cabe ninguna duda de que en todas las aulas de la geografía española se enseñaba y practicaba al máximo el dogma católico, y no solo porque así lo marcaban las leyes, sino también porque los docentes tenían asumidos e interiorizados estos valores religiosos y consideraban que su misión era inculcarlos a las jóvenes generaciones, llegando a extremos como los del maestro de Palma del Río, criticado por la autora de una memoria pedagógica porque "cree obrar perfectamente ocupándose solo de la enseñanza de la Religión que es [lo] único que él considera importante" (Domínguez López, 1955: 49). Sin embargo, sobre la educación patriótica había mucha más disparidad de criterios.

Resulta curioso constatar que el nombre que la Ley de 1945 dio a esta materia, Formación del Espíritu Nacional, no estaba ni mucho menos extendido en la práctica educativa; al menos la mitad de las escuelas la etiquetaban como Formación o Educación Política, dándonos así pistas sobre el sentido que otorgaban a su contenido; en algunos casos se la identificaba directamente con Nacional Sindicalismo, diferenciándosela en este caso de la Educación Patriótica (Llopis Sánchez, 1954: 153-155). Por otra parte, esta asignatura no siempre tenía un hueco bien definido en los horarios o en la distribución de tiempos que las escuelas ofrecían, aunque sí que solía aparecer en el programa de materias. Habitualmente, y salvo casos excepcionales, ocupaba la banda temporal destinada a los contenidos que requerían menos esfuerzo intelectual, a últimas horas de la tarde y en los días cercanos al fin de semana; en ocasiones se integraba con la formación religiosa que, casi con seguridad, le restaría todo el protagonismo; y en un número reducido pero significativo de escuelas, generalmente públicas y privadas de niñas, no aparecía mencionada en los horarios. Sí que se había extendido el nuevo ritual patriótico de iniciar y acabar las clases con las ceremonias en torno a la bandera y el canto de himnos, pero ni siquiera esta práctica era unánime, pues en algunas unitarias, como las de Gran Canaria, los ritos de comienzo de la actividad docente seguían siendo los mismos que en el siglo XIX, "entrada, lista, revista de aseo y oración” (Arias Ramos, 1953: 43).

En muchas escuelas el Frente de Juventudes era el encargado de impartir estas enseñanzas, y en aquellos pueblos donde su presencia era más fuerte - generalmente los más cercanos a Madrid - se intensificaba la implicación de los docentes en estos actos, llegando a 
Políticas educativas y prácticas escolares: la aplicación de la Ley de Enseñanza Primaria de 1945 en las aulas

enviar a la sección local de Falange un "parte mensual de actividades políticas en cuanto a la firmeza, patriotismo del espíritu infantil" (González López, 1955: 37). Por el contrario, en determinadas poblaciones rurales y zonas suburbiales "no se viven apenas las doctrinas del Movimiento" (Marcos Ramos, 1954: 62), y hasta se dejaba entrever sutilmente la oposición de las familias al nuevo régimen, lo que contribuiría a frenar el interés de los docentes por explicar unos temas que les harían significarse políticamente y dificultarían su integración en el entorno. Y la mayoría de los maestros no se sentían preparados ni identificados con los contenidos de esa nueva asignatura, por lo que no podían hacer otra cosa que "limitarse a cantar himnos patrióticos", una situación denunciada por uno de los estudiantes de Pedagogía, Arturo de la Orden Hoz, en su memoria de prácticas, y de la que responsabilizó a "la inconsciencia del Estado de embotellar a niños de diez años la doctrina política de José Antonio, cosa que los niños no comprenden y que a fin de cuentas no pasa de ser la doctrina de un partido" (Orden Hoz, 1952: s.p.).

Algunos docentes sí que intentaron apropiarse y dotar de contenido a la nueva asignatura de Formación del Espíritu Nacional, y la identificaron con la Educación Cívica, una materia que, al igual que la Urbanidad, había estado muy presente en las escuelas españolas, especialmente durante la etapa republicana, pero que jamás había formado parte de ningún currículum oficial. Nuevamente se reprodujeron las dos grandes concepciones que sobre esta materia se habían planteado con anterioridad a la Guerra Civil. Una de ellas la identificaba con la educación patriótica, y buscaba fomentar en los niños los sentimientos de amor a España basados en la grandeza de sus destinos y en su "tradicción [sic] católica" (Arroyo Giralda, 1955: 66, y Yerro Mainar, 1956: 114). La segunda la conceptualizaba como educación para una ciudadanía activa, "la educación para la comprensión del Estado, de su esencia y de sus tareas, y sobre todo, para la voluntad de colaborar como miembro activo en la realización de esas tareas" (Vicente Guillén, 1954: 57). En cualquier caso, aquellos escasos maestros más cercanos a las doctrinas falangistas, y que, en ocasiones, eran los delegados locales del Frente de Juventudes en su pueblo, adaptaron los cuestionarios y programas de Falange a sus propias realidades locales, diseñando ejercicios pensados para despertar los intereses de los alumnos y facilitar el aprendizaje significativo (Guzmán Cebrián, 1956: 96-100).

\section{Los Cuestionarios Nacionales de Enseñanza Primaria de 1953 en las aulas: aspectos metodológicos}

La publicación de los Cuestionarios Nacionales de 1953 tuvo un impacto inmediato en la práctica escolar, y esta realidad aparece reflejada muy claramente en las memorias pedagógicas. Así, las escritas con anterioridad a 1954 manifestaban que las escuelas estudiadas poseían unos cuestionarios, casi siempre elaborados por la Inspección Provincial, que se aplicaban muy parcialmente por no adaptarse a la realidad local y que permanecían inmutables a lo largo de varios años (Nieto, 1953: 53-54). Algunos maestros rechazaban estos cuestionarios "por ser muy difíciles y demasiado extensos", y entendían como programa "el que figura en los distintos libros de texto" (León Carrasco, 1950: 18). Sin embargo, desde el momento en el que los Cuestionarios de 1953 entraron en vigor, todos los docentes proclamaron que los seguían fielmente, si bien reconocían haberlos flexibilizado, adaptado y sintetizado en un programa mínimo que, a pesar de ser mucho más reducido, era asimilado solamente por el 30\% de la población escolar (Arroyo Giralda, 1955: 63 y Lago Mauricio, 1956: 27-28).

La mayoría de las experiencias escolares daban testimonio de unas prácticas enraizadas en la pedagogía decimonónica, con el uso y abuso de recursos tan antañones como las lecciones a coro, el empleo de monitores y ayudantes, el 
"dar o tomar" las lecciones tras su aprendizaje memorístico, las lecturas en corro o la corrección de dictados en la pizarra. La pervivencia de estas prácticas, rechazadas tanto por las reformas escolares republicanas como por las orientaciones técnicas franquistas, dan idea del arraigo de lo que Tyack y Cuban han dado en llamar la "gramática de la escuela", es decir, la pervivencia de una cultura escolar impermeable a cualquier tipo de reformas y que funciona con sus propias pautas de continuidad a lo largo del tiempo.

Frente a este modelo tradicional las memorias dan testimonio de un pequeño porcentaje de docentes que realizaban ensayos más novedosos, dirigidos a estimular el interés de los alumnos. Entre estas experiencias podemos encontrar muchas actividades similares a las expuestas en los Cuestionarios de 1953, tales como los viajes imaginarios y la construcción de mapas en relieve para la enseñanza de la Geografía, el uso de biografías para la Historia, las narraciones y conversaciones sobre temas de la vida cotidiana, los paseos y excursiones para las Ciencias de la Naturaleza, las clases al aire libre, los periódicos escolares, el cuidado de las macetas en las clases de niñas y de los animales en las de niños, los trabajos en grupos, los cuentos dramatizados, los periódicos murales, los materiales didácticos autoconstruidos, las actividades manuales para la fijación de los contenidos intelectuales, las experiencias de autogobierno aplicadas a la recaudación de dinero para la Santa Infancia o a la organización de la biblioteca escolar, los cuadernos sobre temas de actualidad o sobre cuestiones monográficas investigadas por los propios alumnos y las prácticas de jardinería y horticultura. En una primera aproximación, podría afirmarse que la tímida aparición de todos estos ensayos fue una consecuencia directa de la publicación de los Cuestionarios Nacionales de 1953.

Sin embargo, nuestra conclusión es otra muy diferente, y se sustenta en la hipótesis de continuidad anteriormente mencionada. Nosotras creemos que estas actividades representaban la pervivencia de un modelo renovador de la enseñanza primaria lentamente construido en los años veinte y treinta y que reflejaba la apropiación que del movimiento de la Nueva Educación se hizo en las escuelas españolas. La bisagra central de ese modelo era la sustitución del concepto de asignatura por el de actividad, y el cambio de las lecciones por actividades interrelacionadas en torno a contenidos comunes, según la metodología decrolyana. Los centros de interés acabaron reducidos al denominado "tema del día", alrededor del cual giraba la labor cotidiana (Pozo Andrés, 2007: 162), y esta idea se mantuvo en algunas escuelas de los años cuarenta y cincuenta, que afirmaban no querer organizar un horario con distribución de materias, "por estimar que hemos de explicar un tema diario sin mezcla de otro alguno" (Guzmán Cebrián, 1956: 92). La colección de actividades mencionadas, y otras muchas, eran insignias y símbolos de lo que se consideraba la modernidad pedagógica $\mathrm{y}$, por eso, convenientemente desideologizadas, pervivieron, también como signos de modernidad, durante el régimen franquista. Y sus mantenedores fueron aquellos educadores, docentes e inspectores, que habían sido formados en esa cultura pedagógica de la renovación. Las memorias de prácticas nos ofrecen algunos ejemplos que justifican nuestra afirmación.

Así, el grupo escolar de Son Espanyolet en Palma de Mallorca, cuyo director —aunque en la monografía no se recogiera su nombre- era Miquel Deyá Palerm, conocido maestro freinetista antes de la Guerra Civil (Jaume Campaner, 2001)2 , seguía un elaborado sistema de autogobierno, con delegación de responsabilidades entre los diferentes niños de la escuela; planteaba actividades cooperativas denominadas indirectamente "proyectos" y que crearon no poca expectación en la sociedad balear; pretendía fundir todas las asignaturas "en el común denominador de España”; y había abolido la obligatoriedad de comulgar en la misa sabatina, para evitar violentar "la conciencia 
del niño", una iniciativa que llenó de dudas al propio autor de la memoria (Enseñat Lázaro, 1952). En Banyoles había recalado Lluis G. Bover Oliveras, otro maestro freinetista y compañero de Patricio Redondo Moreno en 193536. Este docente había adaptado todo tipo de instituciones utilizadas en los años treinta para la educación moral, como las sociedades cooperativas o las Ligas de Bondad, que aplicaba en los años cincuenta para estimular las donaciones a la Santa Infancia y para extender los preceptos dominicales. Y también llevó una pequeña imprenta - parece ser que fabricada por él mismo- con la que los niños elaboraron el periódico Ofrenda, cuyo primer número salió en junio de 1951 y que posiblemente fuera el primer ensayo Freinet realizado después de la Guerra Civil aunque, por supuesto, no se citaba al pedagogo francés, quedando el propio Bover como inventor de la técnica. Desde luego, se mantenía el espíritu inspirador de la misma, expresado en la redacción de un alumno: "Para confeccionar 'nuestro periódico' se escogen las composiciones originales más interesantes, entre las que han escrito los niños sobre diversos temas. Dichos escritos son corregidos por el maestro o por los niños, en colaboración" (Julbe Roda, 1951: s.p.). No es de extrañar que la mayoría de los artículos recogidos en este primer número reflejaran visitas de los alumnos a la parroquia o estancias en los campamentos del Frente de Juventudes. La imprenta en la escuela surgió para que el niño se expresase libremente sobre su vida cotidiana, y la vida de un niño en los años cincuenta giraba en torno a la Iglesia y a la Falange.

\section{Notas}

${ }^{1}$ Este artículo se ha realizado en el marco del Proyecto de I+D+i "Origen y desarrollo del conocimiento teórico de la educación en España", EDU2010-17367.

${ }^{2}$ Agradecemos a Xisca Comas y al grupo de investigación de Historia de la Educación de les Illes Balears la pista y los datos que nos proporcionaron sobre este maestro mallorquín.

\section{Referencias bibliográficas}

Arias Ramos, $M^{a}$ T. (1953). La enseñanza en Firgas, Gran Canaria: FRM 443. Museo de Historia de la Educación "Manuel Bartolomé Cossío", Universidad Complutense de Madrid (a partir de ahora MBC, UCM).

Arroyo Giralda, C. (1955). Problemas educativos en Navelgas (Asturias). FRM 795. MBC, UCM.

Beltrán EsCRig, J. (1956). Lucena del Cid: Sus problemas de orden educativo, Castellón: FRM 328. MBC, UCM.

Bernal, Ma C. (1953). La enseñanza en Arjonilla (Jaén). FRM 673. MBC, UCM.

BerRio, J. R.; RABAZAS, T. y RAmOS, S. (2006). The reception of the New Education in Spain by means of Manuals on the History of Education for Teacher Training Colleges (1898-1976), Paedagogica Historica, 42 (1-2), 127-141.

Colmenar Orzaes, C. (2012). El Fondo Romero Marín del Museo Manuel Bartolomé Cossío. Memorias sobre las prácticas escolares: la educación infantil. En P.L. Moreno Martínez y A. Sebastián Vicente (eds.), Patrimonio y etnografía de la escuela en España y Portugal durante el siglo XX. Murcia: SEPHE-CEME-Universidad de Murcia, 197-210.

Cuesta Fernández, R. (1997). Sociogénesis de una disciplina escolar: la historia. Barcelona: PomaresCorredor (versión electrónica en 2009: http://www.nebraskaria.es/Nebraskaria/Trabajos_y_publicaciones_files/Socioge\%CC\%81nesis...\%20.pdf) 
Domínguez López, S. (1955). El problema pedagógico en Palma del Río. Córdoba: FRM 406. MBC, UCM.

Enseñat Lázaro, A. (1952). Grupo escolar de Son Españolet. Palma de Mallorca: FRM 261. MBC, UCM.

GalÁn Martínez, E. (1953). La cultura en Sayago. Zamora: FRM 159. MBC, UCM.

GonZÁlez López, F. (1955). La enseñanza en Aranjuez y sus problemas. Madrid: FRM 565. MBC, UCM.

Guzmán Cebrián, J. (1956). Estudio monográfico de los problemas educativos de Villarta de San Juan. Ciudad Real: FRM 178. MBC, UCM.

Jaume Campaner, M. (2001). Freinet a Mallorca. Miquel Deyà Palerm i l'escola de Consell (19301940). Palma de Mallorca: Lleonard Muntaner Editor.

Julbe Roda, F. (1951). Cómo hacemos nuestro periódico. Ofrenda, 1, s.p.

Lago Mauricio, Ma R. (1956). Algunos problemas escolares en la Isla de Arosa (Pontevedra). FRM 040. MBC, UCM.

LeÓn CARRAsco, Ma L. (1950). Monografía pedagógica. La enseñanza en Morata de Tajuña. Madrid: FRM 244. MBC, UCM.

Ley (1945) de 17 de julio sobre Educación Primaria. Boletín Oficial del Estado, 199, 18 de julio de $1945,385-416$.

Llopis SÁnCHEZ, J. (1954). Problemas pedagógicos de Albalate de la Ribera, Valencia: FRM 192. MBC, UCM.

López BAusela, J. R. (2012). Los programas escolares inéditos de 1938 en la España de Franco: el cerco pedagógico a la modernidad. Madrid: UNED y PubliCan.

López Chillón, R. y García De Leza, D.M. (1953). "Hijas de Jesús”. La labor educativa del Instituto de las RR Hijas de Jesús. FRM 123. MBC, UCM.

LóPEZ DEl CASTILlo, M M $^{\mathrm{T}}$ T. (2013). Función Orientadora de la Inspección: cuestionarios y programas antes de 1953. Supervisión 21, 27, 1-8. http://usie.es/SUPERVISION21/2013_27/ARTCLS_Elaboracuestinspecprovinc.pdf

Maillo García, A. (1989). Historia crítica de la Inspección escolar en España. Madrid: Josmar.

Mainer Baqué, J. y Mateos Montero, J. (2011). Saber, poder y servicio. Un pedagogo orgánico del Estado: Adolfo Maillo. Valencia: Tirant lo Blanch.

Marcos Ramos, A. (1954). Problemas pedagógicos de Berlanga de Duero. Soria: FRM 233. MBC, UCM.

Martínez Navarro, A. (1997). Un seminario sobre fuentes históricas en el Museo de Historia de la Educación de la Facultad de Educación de la Universidad Complutense. Revista Complutense de Educación, 8 (1), 305-318.

Ministerio de EduCACión Nacional (1953). Cuestionarios Nacionales para la enseñanza primaria. Madrid: Dirección General de Enseñanza Primaria. Servicio de Publicaciones del Ministerio de Educación Nacional.

Navamuel Isasi, Ma P. (1954). El magisterio de la Iglesia. “Fray Luis de León”. Valladolid: FRM 830. MBC, UCM.

NieTO, B. (1953). Bujalance y su problemática cultural. Córdoba: FRM 61. MBC, UCM.

Orden Hoz, A. de la (1952). La enseñanza en Campillo de Aranda. Burgos: FRM 292. MBC, UCM.

Peralta Ortiz, Ma D. (1996). El maestro de la España nacional (1936-1975). Tesis doctoral inédita. Madrid: UNED.

Pericacho Gómez, F. J. (2012). El Fondo Romero Marín del Museo "Manuel Bartolomé Cossío". Documentos sobre las prácticas escolares: la labor pedagógica de la ciudad de los muchachos de Vallecas. En P. L. Moreno Martínez y A. Sebastián Vicente (eds.), Patrimonio y etnografía de la 
Políticas educativas y prácticas escolares: la aplicación de la Ley de Enseñanza Primaria de 1945 en las aulas

escuela en España y Portugal durante el siglo XX. Murcia: SEPHE-CEME-Universidad de Murcia, 311-322.

Poveda Sanz, M. y Rabazas Romero, T. (2012). El Fondo Romero Marín del Museo "Manuel Bartolomé Cossío". Análisis de las memorias de las prácticas de Pedagogía. En P. L. Moreno Martínez y A. Sebastián Vicente (eds.), Patrimonio y etnografía de la escuela en España y Portugal durante el siglo XX. Murcia: SEPHE-CEME-Universidad de Murcia, 323-336.

Pozo AndrÉs, Ma M. DEL (2007). Desde l'Ermitage a la escuela rural española: Introducción, difusión y apropiación de los "centros de interés" decrolyanos (1907-1939), Revista de Educación, $\mathrm{n}^{\circ}$ extr., 143-166.

Pozo Andrés, Ma M. DEl y RABAzAs Romero, T. (2010). Imatges fotogràfiques i cultura escolar en el franquisme: una exploració de l'arxiu etnogràfic, Educació i Historia, 15, 165-194.

Pozo Andrés, Ma M. Del y Rabazas Romero, T. (2011). Classroom Cultures. Researching the Pioneer Ethnographical Researches (Madrid, 1950-1970). En S. Braster, I. Grosvenor y Ma M. DEL Pozo Andrés (eds.), The Black Box of Schooling. A Cultural History of the Classroom. Bruselas: Peter Lang, 99-120.

Puelles Benítez, M. DE (1999). Educación e Ideología en la España Contemporánea. Madrid: Tecnos. Rabazas Romero, T.; Ramos Zamora, S. y Ruiz Berrio, J. (2009). La evolución del material escolar a través de los manuales de Pedagogía (1875-1936). Revista Española de Pedagogía, 243, 275298.

Ramos Zamora, S. (2012). Labor pedagógica de los Hogares de Auxilio Social a través de las memorias del Fondo Romero Marín del Museo "Manuel Bartolomé Cossío". En P. L. Moreno Martínez y A. Sebastián Vicente (eds.), Patrimonio y etnografía de la escuela en España y Portugal durante el siglo XX. Murcia: SEPHE-CEME-Universidad de Murcia, 337-354.

Selles Vercher, J. (1956). El analfabetismo en Sueca desde 1900 a 1950. Valencia: FRM 372. MBC, UCM.

Vicente Guillén, A. (1954). La pedagogía y sus problemas en un rincón del pirineo catalán. Lleida: FRM 690. MBC, UCM.

YerRo Mainar, M. Del (1956). Labor pedagógica realizada por el grupo escolar del Santo Ángel. Madrid: FRM 054. MBC, UCM.

\section{Abstract}

Educational Politics and School Practices: The Implementation of the 1945 Primary Education Act in the Classrooms

INTRODUCTION. This article analyses the influence of educational politics in the school practice during the first years of Francoism. We study the impact of some educational principles established in the 1945 Primary Education Act and in the 1953 national Syllabuses for Primary Education on the daily life of schools during the 1950's. METHOD. The article combines the historical method for the analysis of educational politics and the ethnographic method for the interpretation of school practices. The main source of documentation has been the collection of pedagogical reports archived in the Collection "Anselmo Romero Marín" preserved in the Museum "M.B. Cossío" from the Complutense University. RESULTS. The analysis that has been carried out allows us to detect several continuities with the curricular approaches of previous historical periods and with the methodological renovation of the 1930's. On the other hand, it does not seem that the educational legislation of the first Francoist period was very present in the daily school life, except for the most 
symbolic and ritualized aspects. This conclusion leads us to confirm that the history of the classroom is built from the empirical school culture developed by schoolteachers with which we cannot find any significant breaks despite the clear ideological changes observed in educational politics. DISCUSSION. A possible line of discussion in the historiographical debate about Francoism could arise from introducing the theoretical model of the "grammar of schooling" developed several years ago by Tyack and Cuban. The discussion might then focus on how far Francoist educational politics entailed a curricular and methodological break with the previous historical period. On the one hand we could state that educational practices are immune to political changes and, on the other hand we could say that they are dependent of the mentality of teachers and of their professional dynamics.

Key words: Primary education, Francoism, Primary Education Act of 1945, Educational politics.

\section{Résumé}

Les politiques éducatives et les pratiques scolaires: l'application de la Loi d'Enseignement Primaire de 1945 aux salles de classe

INTRODUCTION. Cet article analyse l'influence de la politique éducative dans le milieu scolaire du premier franquisme. Nous étudions l'influence de quelques principes éducatifs énoncés dans la Loi d'Enseignement Primaire de 1945, sur les pratiques scolaires et sur les questionnaires nationaux pour l'enseignement primaire qui ont été approuvés en 1953. MÉTHODE. L'article combine la méthode de l'histoire de l'éducation pour l'analyse de la politique éducative avec la méthode ethnographique pour l'interprétation des pratiques scolaires. La principale source de documentation a été la collection des mémoires pédagogiques qui font partie du Fond "Anselmo Romero Marín" conservé au Musée "M. B. Cossío" de l'Université Complutense. RÉSULTATS. L'analyse nous a permis de détecter certaines continuités avec les approches pédagogiques propres de périodes historiques précédentes aussi bien qu'avec les méthodes de rénovation des années trente. D’autre part, il ne semble pas que la législation éducative du premier franquisme soit très présente dans la vie quotidienne de l'école, sauf dans des aspects de caractère symbolique et ritualisé. Ceci nous a permis de confirmer que l'histoire de l'école se construit avec la culture scolaire empirique que les enseignants développent et que, malgré les virages idéologiques que la politique de l'éducation puisse souffrir, on n'aperçoit pas de sauts significatifs. DISCUSSION. Il serait possible d'introduire dans le débat historiographique sur le franquisme le modèle de la "grammaire de l'école", développée il y a quelques années par Tyack et Cuban. La discussion pourrait porter sur la question de la mesure dans laquelle la politique éducative du franquisme représente une rupture avec le curriculum et les méthodes d'enseignement de l'étape précédente. À certains égards, il pourrait être interprété que les pratiques pédagogiques sont à l'abri des changements politiques et néanmoins, plus dépendantes de la mentalité et les dynamiques professionnelles des enseignants et des enseignantes.

Mots clés: Enseignement primaire, Franquisme, Loi de 1945, Politique éducative. 


\section{Perfil profesional de las autoras}

\section{María del Mar del Pozo Andrés}

Catedrática de Teoría e Historia de la Educación de la Universidad de Alcalá. Es Doctora en Filosofía y Ciencias de la Educación por la UCM con Premio Extraordinario de Doctorado. Sus líneas principales de investigación son la recepción en España de los movimientos internacionales de renovación pedagógica y la etnohistoria de la escuela, especialmente el análisis de imágenes en la historiografía educativa. Su obra más reciente es Justa Freire o la pasión de educar. Biografía de una maestra atrapada en la historia de España (1896-1965) (Octaedro, 2013).

Correo electrónico de contacto: mar.delpozoandres@gmail.com

\section{Teresa Rabazas Romero}

Profesora titular de Universidad del Departamento de Teoría e Historia de la Educación de la Universidad Complutense de Madrid. Es Doctora en Filosofía y Ciencias de la Educación por la UCM con la obtención de Premio Extraordinario de Doctorado. Ha desarrollado como líneas principales de investigación el análisis de los manuales como objeto y fuente de conocimiento de la cultura escolar en la España contemporánea, así como el análisis del currículo desde una perspectiva de género. Correo electrónico de contacto: rabarom@edu.ucm.es 